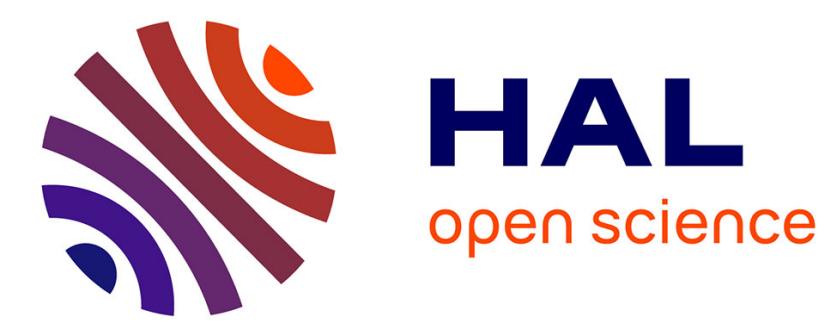

\title{
Croissance de couches de Si1-xGex par réaction chimique a partir d'une phase gazeuse: étude thermodynamique et analyse du transfert de matière
}

H. Rouch, M. Pons, C. Bernard, R. Madar

\section{- To cite this version:}

H. Rouch, M. Pons, C. Bernard, R. Madar. Croissance de couches de Si1-xGex par réaction chimique a partir d'une phase gazeuse : étude thermodynamique et analyse du transfert de matière. Journal de Physique III, 1995, 5 (6), pp.759-773. 10.1051/jp3:1995159 • jpa-00249344

HAL Id: jpa-00249344

https://hal.science/jpa-00249344

Submitted on 1 Jan 1995

HAL is a multi-disciplinary open access archive for the deposit and dissemination of scientific research documents, whether they are published or not. The documents may come from teaching and research institutions in France or abroad, or from public or private research centers.
L'archive ouverte pluridisciplinaire HAL, est destinée au dépôt et à la diffusion de documents scientifiques de niveau recherche, publiés ou non, émanant des établissements d'enseignement et de recherche français ou étrangers, des laboratoires publics ou privés. 
Classification

Phyśics Abstracts

$81.15 \mathrm{H}-47.70 \mathrm{~F}$

\title{
Croissance de couches de $\mathbf{S i}_{1-x} \mathbf{G e}_{x}$ par réaction chimique à partir d'une phase gazeuse : étude thermodynamique et analyse du transfert de matière
}

\author{
H. Rouch $\left({ }^{1}\right)$, M. Pons $\left({ }^{1}\right)$, C. Bernard $\left({ }^{2}\right)$ et R. $\operatorname{Madar}\left({ }^{3}\right)$ \\ ( $\left.{ }^{1}\right)$ S2MC, URA n ${ }^{\circ}$ 413/ENSEEG/INPG, BP 75, Domaine Universitaire, 38402 Saint-Martin \\ d'Hères, France \\ $\left({ }^{2}\right)$ LTPCM, URA n ${ }^{\circ}$ 29/ENSEEG/INPG, BP 75, Domaine Universitaire, 38402 Saint-Martin \\ d'Hères, France \\ $\left({ }^{3}\right)$ LMGP, URA $n^{\circ}$ 1109/ENSPG/INPG, BP 46, Domaine Universitaire, 38402 Saint-Martin \\ d'Hères, France
}

(Reçu le 10 novembre 1994, révisé le 15 mars 1995, accepté le 21 mars 1995)

Résumé. - La croissance de couches de $\mathrm{Si}_{1-x} \mathrm{Ge}_{x}$ à haute température $(1300 \mathrm{~K})$ et basse pression $(315 \mathrm{~Pa})$ par réaction chimique à partir d'une phase gazeuse a été étudiée à l'aide d'une approche thermodynamique et de l'analyse du transfert de matière pour laquelle des hypothèses très simplificatrices ont été émises. Les résultats ont montré que l'analyse thermodynamique, seule, ne permet pas d'interpréter les mesures concernant la composition du matériau déposé. Quant à l'étude du transfert de matière, elle a montré que la thermodiffusion jouait un rôle important sur la composition du matériau déposé ainsi que sur l'uniformité du dépôt sur des substrats de grandes dimensions.

\begin{abstract}
The growth of $\mathrm{Si}_{1-x} \mathrm{Ge}_{x}$ layers at a high temperature $(1300 \mathrm{~K})$ and low pressure (315 $\mathrm{Pa}$ ) (by chemical vapour deposition) was studied by a thermodynamic approach and a mass transport analysis using simplifying assumptions. The results showed that the thermodynamic analysis does not permit alone to explain the experimental measurements concerning the composition of the layer. The mass transport analysis showed that thermodiffusion would be of importance on layer composition and uniformity for large substrates.
\end{abstract}

\section{Introduction}

Les couches de $\mathrm{Si}_{1-x} \mathrm{Ge}_{\boldsymbol{x}}$ sont utilisées en microélectronique [1,2], dans l'élaboration de cristaux de monochromateurs [3] et pourraient être utilisées pour des composants optiques. Des revêtements de $\mathrm{Si}_{1-x} \mathrm{Ge}_{x}$ ont été élaborés, dans des conditions expérimentales (pression et température) très variées et avec différents types de réacteurs. La gamme de pression s'étend de l'ultra-vide pour le procédé MBE (Molecular Beam Epitaxy) à la pression atmosphérique 
Tableau I. - Espèces gazeuses utilisées dans le modèle (les données thermodynamiques des espèces gazeuses écrites en italıque ont été estimées).

[Gaseous species used for the model (thermodynamic data of gaseous species written in italic were estimated.]

\begin{tabular}{|c|c|c|c|c|c|c|c|}
\hline $\mathrm{SiH}_{4}$ & $\mathrm{SiH}_{3}$ & $\mathrm{SiH}_{2}$ & $\mathrm{SiH}$ & $\mathrm{SiCl}_{4}$ & $\mathrm{SiCl}_{3}$ & $\mathrm{SiCl}_{2}$ & $\mathrm{SiCl}$ \\
\hline $\mathrm{GeH}_{4}$ & $\mathrm{GeH}_{3}$ & $\mathrm{GeH}_{2}$ & $\mathrm{GeH}$ & $\mathrm{GeCl}_{4}$ & $\mathrm{GeCl}_{3}$ & $\mathrm{GeCl}_{2}$ & $\mathrm{GeCl}$ \\
\hline $\mathrm{Si}_{2} \mathrm{H}_{6}$ & $\mathrm{Si}_{2} \mathrm{Cl}_{6}$ & $\mathrm{Si}_{1}$ & $\mathrm{SiHCl}_{3}$ & $\mathrm{SiH}_{2} \mathrm{Cl}_{2}$ & $\mathrm{SiH}_{3} \mathrm{Cl}$ & & \\
\hline $\mathrm{Ge}_{2} \mathrm{H}_{6}$ & $\mathrm{Ge}_{2} \mathrm{Cl}_{6}$ & $\mathrm{Ge}_{1}$ & $\mathrm{GeHCl}_{3}$ & $\mathrm{GeH}_{2} \mathrm{Cl}_{2}$ & $\mathrm{GeH}_{3} \mathrm{Cl}$ & & \\
\hline
\end{tabular}

pour le procédé CVD (Chemical Vapor Deposition) conventionnel en passant par des pressions réduites pour les procédés CVD à chauffage rapide par lampes.

L'étude expérimentale concernant la croissance de couches de $\mathrm{Si}_{1-x} \mathrm{Ge}_{x}$ par réaction chimique à partir d'une phase gazeuse (CVD) a déjà été développée par Madar et al. [3]. Cette étude, réalisée pour une température extrême $(1300 \mathrm{~K})$ et à basse pression $(133 \mathrm{~Pa})$ afin d'obtenir des vitesses de croissance élevées (de l'ordre de $1 \mu \mathrm{m} . \mathrm{mn}^{-1}$ ) et d'éviter les phénomènes de nucléation homogène dans le réacteur, avait révélé que la composition de la couche de $\mathrm{Si}_{1-x} \mathrm{Ge}_{x}$ était très sensible à des modifications de géométrie du réacteur, de dimension du substrat, aux variations de débit et à la nature du mélange gazeux réactif. Pour interpréter ces faits, dans le présent article, une étude thermodynamique et une étude de transfert de matière sont développées. Elles ont pour but d'appréhender l'importance relative des phénomènes physiques et chimiques mis en jeu lors de la croissance de $\mathrm{Si}_{1-x} \mathrm{Ge}_{x}$.

\section{Approche thermodynamique}

L'approche thermodynamique a pour but de dégager l'influence des gaz réactifs sur la composition du revêtement par une analyse des réactions homogènes et hétérogènes. Cette approche suppose un système fermé et l'équilibre thermodynamique atteint. Elle ne peut rendre compte des gradients de composition et donc de la composition réelle du mélange gazeux au voisinage du substrat.

Les paramètres de l'équilibre sont calculés par minimisation de l'énergie totale de Gibbs du système chimique [4]. Cette approche a été appliquée, à la température de $1300 \mathrm{~K}$, aux mélanges (a) $\mathrm{SiH}_{4}$ et $\mathrm{GeH}_{4}$ dilués dans l'argon, (b) $\mathrm{SiH}_{4}, \mathrm{GeH}_{4}$ et $\mathrm{HCl}$ dilués dans l'argon et l'hydrogène, (c) $\mathrm{SiH}_{4}$ et $\mathrm{GeCl}_{4}$ dilués dans l'argon.

2.1. LES DONNÉES. - Les calculs thermodynamiques ont été réalisés à l'aide du logiciel Mélange [4] connecté avec la banque de donnée SGTE (Scientific Group Thermodata Europe) [5]. Les espèces gazeuses qui ont été prises en compte pour ces calculs sont répertoriées dans le tableau I. La phase solide envisagée est la solution solide $\mathrm{Si}_{1-x} \mathrm{Ge}_{x}$. Pour le système $\mathrm{Ge}-\mathrm{H}-\mathrm{Cl}$, toutes les données nécessaires n'étaient pas disponibles. Elles ont été estimées par des méthodes de pondération pour la famille de composés $\mathrm{GeHCl}_{3}, \mathrm{GeH}_{2} \mathrm{Cl}_{2}$ et $\mathrm{GeH}_{3} \mathrm{Cl}$ et en supposant une similitude de comportement des éléments $\mathrm{Ge}$ et $\mathrm{Si}$ vis-à-vis des atomes $\mathrm{H}$ et $\mathrm{Cl}$ pour les autres espèces gazeuses manquantes $\left(\mathrm{GeH}, \mathrm{GeH}_{2}, \mathrm{GeH}_{3}\right)$ [6]. 
Tableau II. - Données thermochimiques pour le système Ge-H-Cl $\left(C_{p}=A+B T+C T^{2}+D T^{-2}\right.$ en J.mol ${ }^{-1}$ ) [6] non décrites dans la banque SGTE [5].

[Thermodynamic data for the Ge-H-Cl system $\left(C_{p}=A+B T+C T^{2}+D T^{-2}\right.$ in J.mol $\left.{ }^{-1}\right)$ [6] not described in the SGTE databank [5].]

\begin{tabular}{|l|c|c|c|c|c|c|}
\cline { 2 - 7 } \multicolumn{1}{c|}{} & $\Delta H_{298}$ & $S_{298}$ & $A$ & $B$ & $C$ & $D$ \\
\hline $\mathrm{GeH}$ & $3,0355 \times 10^{+5}$ & $2,1299 \times 10^{+2}$ & $2,9142 \times 10^{+1}$ & $7,2249 \times 10^{-3}$ & $-2,4130 \times 10^{-7}$ & $3,4411 \times 10^{+6}$ \\
\hline $\mathrm{GeH}_{2}$ & $2,3263 \times 10^{+5}$ & $2,2200 \times 10^{+2}$ & $4,0327 \times 10^{+1}$ & $1,234 \times 10^{-2}$ & $-1,6269 \times 10^{-7}$ & $1,5737 \times 10^{+6}$ \\
\hline $\mathrm{GeH}_{3}$ & $1,6171 \times 10^{+5}$ & $2,2999 \times 10^{+2}$ & $5,1531 \times 10^{+1}$ & $1,7232 \times 10^{-2}$ & $-8,4219 \times 10^{-8}$ & $-2,9369 \times 10^{+5}$ \\
\hline $\mathrm{GeHCl}_{3}$ & $-3,5230 \times 10^{+5}$ & $3,2900 \times 10^{+2}$ & $9,5814 \times 10^{-3}$ & $6,1010 \times 10^{-3}$ & $1,5529 \times 10^{-8}$ & $-1,2832 \times 10^{+6}$ \\
\hline $\mathrm{GeH}_{2} \mathrm{Cl}_{2}$ & $-2,0460 \times 10^{+5}$ & $3,0199 \times 10^{+2}$ & $8,4788 \times 10^{+1}$ & $1,1439 \times 10^{-2}$ & $8,4630 \times 10^{-9}$ & $-1,5758 \times 10^{+6}$ \\
\hline $\mathrm{GeH}_{3} \mathrm{Cl}$ & $-5,6910 \times 10^{+4}$ & $2,6599 \times 10^{+2}$ & $7,3761 \times 10^{+1}$ & $1,6777 \times 10^{-2}$ & $1,3963 \times 10^{-1}$ & $-1,3732 \times 10^{+6}$ \\
\hline
\end{tabular}

Le tableau II résume les données thermodynamiques non décrites dans la banque SGTE. Les méthodes d'estimation utilisées pourraient conduire à des valeurs approchées de la composition à l'équilibre. Heureusement, comme on le verra par la suite, les espèces gazeuses concernées n'ont pas un rôle primordial dans l'établissement de l'équilibre. Pour le calcul de l'énergie libre de Gibbs de formation de la solution solide $\mathrm{Si}_{1-x} \mathrm{Ge}_{x}$, et de la phase liquide correspondante, Bergman et al. [7] ont ajusté un modèle de l'énergie libre de Gibbs sur les résultats de leurs mesures calorimétriques.

Comme la solution solide $\mathrm{Si}_{1-x}-\mathrm{Ge}_{x}$ et la phase liquide ont un comportement de solution strictement régulière, l'énergie totale de Gibbs d'excès est donnée par [7] :

$$
\Delta G_{\mathrm{f}}^{x \mathrm{~s}}=\alpha X(1-X)
$$

pour le solide $\quad \alpha_{s}=3500 \mathrm{~J} . \mathrm{mole}^{-1}$

pour le liquide $\alpha_{1}=6500 \mathrm{~J} \cdot \mathrm{mole}^{-1}$

2.2. Simulation thermodynamique. - Pour les calculs thermodynamiques, la pression totale, la température et les pressions partielles (ou concentrations) initiales des différentes espèces gazeuses doivent être connues. Les paramètres $X, Y$ et $\mathrm{Cl} / \mathrm{H}$ seront utilisées pour caractériser les systèmes simulés :

$$
X=\frac{n_{\mathrm{Ge}}}{n_{\mathrm{Ge}}+n_{\mathrm{Si}}}
$$

où $n_{\mathrm{Ge}}$ et $n_{\mathrm{Si}}$ représentent le nombre de moles de germanium et de silicium dans la couche de $\mathrm{Si}_{1-x} \mathrm{Ge}_{x}$.

$$
Y=\frac{n_{\mathrm{GeH}_{4}}^{0}}{n_{\mathrm{GeH}_{4}}^{0}+n_{\mathrm{S}_{1} \mathrm{H}_{4}}^{0}} \text { ou } Y=\frac{n_{\mathrm{GeCl}_{4}}^{0}}{n_{\mathrm{GeCl}_{4}}^{0}+n_{\mathrm{SiH}_{4}}^{0}}
$$

où les termes $n^{0}$ représentent les nombres de moles de gaz réactifs à l'entrée du réacteur. $Y$ représente le rapport du nombre d'atomes de germanium sur le nombre d'atomes de germanium et de silicium introduits dans le réacteur. La grandeur $\mathrm{Cl} / \mathrm{H}$ est définie comme le rapport 


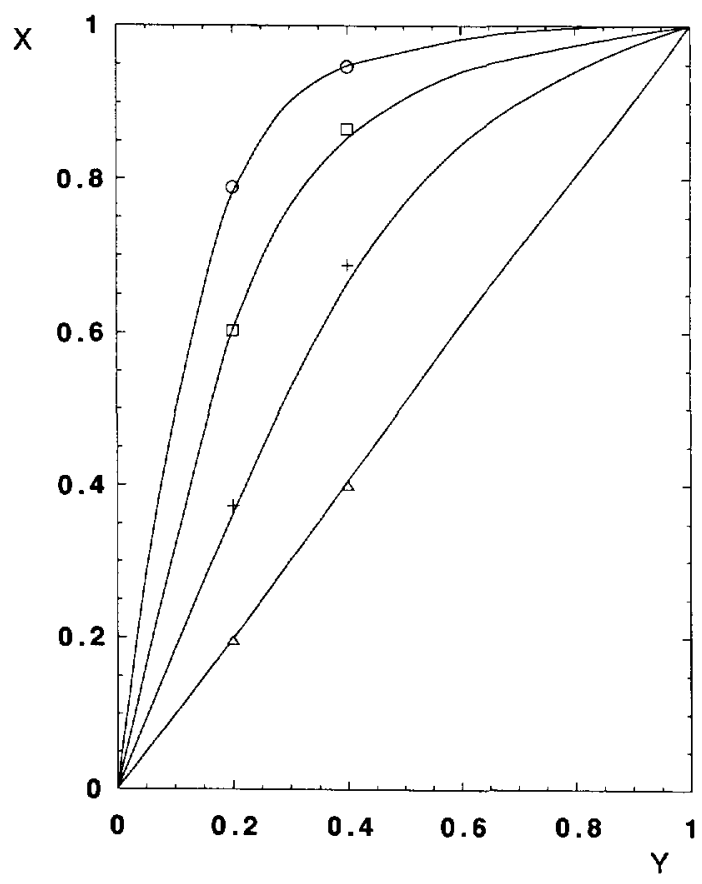

Fig. 1. - Composition à l'équilibre de la phase solide $\mathrm{Si}_{1-x} \mathrm{Ge}_{x}$ en fonction de la composition $(Y)$ du mélange $\mathrm{SiH}_{4}-\mathrm{GeCl}_{4}-\mathrm{H}_{2}$ introduit et pour 4 rapports $\mathrm{Cl} / \mathrm{H}$ à $1200 \mathrm{~K}$ et $105 \mathrm{~Pa}$. ( - ) résultats de Tang et al. [9]. $(\mathrm{O}, \square,+, \Delta)$ : résultats de cette étude pour $\mathrm{Cl} / \mathrm{H}=0,1 ; 0,05 ; 0,02 ; 0,001$.

[Composition of the $\mathrm{Si}_{1-x} \mathrm{Ge}_{x}$ solid phase as a function of the composition $(Y)$ of the mixture $\left(\mathrm{SiH}_{4}-\right.$ $\mathrm{GeCl}_{4}-\mathrm{H}_{2}$ ) at equilibrium for $4 \mathrm{Cl} / \mathrm{H}$ ratio at $1200 \mathrm{~K}$ and $105 \mathrm{~Pa}$. (- ) Tang et al. [9] results $(\mathrm{O}, \square,+, \Delta)$ results of this study for $\mathrm{Cl} / \mathrm{H}=0.1 ; 0.05 ; 0.02 ; 0.001$.]

du nombre d'atomes de chlore sur le nombre d'atomes d'hydrogène injectés dans le réacteur. Comme les résultats de calculs de l'équilibre thermodynamique dans le système $\mathrm{SiH}_{4}-\mathrm{GeCl}_{4}-\mathrm{H}_{2}$ à pression atmosphérique ont déjà été publiés par Chang et al. [8] puis par Tang et al. [9], nous avons utilisé ce mélange afin de déterminer la validité de notre base de donnée en comparant nos résultats à ceux obtenus par Tang et al. [9]. Bien que de petites différences aient été notées, comme par exemple, la présence de $\mathrm{SiCl}_{3}$ à l'équilibre, espèce non prise en compte par Tang et al. [9], l'accord entre les deux études est excellent (Fig. 1).

Pour le système $\mathrm{SiH}_{4}-\mathrm{GeH}_{4}-\mathrm{Ar}$, les calculs ont été effectués pour les gammes de pression : $10^{2}-10^{5} \mathrm{~Pa}$, de température : $600-1400 \mathrm{~K}$, de dilution de $\mathrm{GeH}_{4}$ et $\mathrm{SiH}_{4}$ dans l'argon : $1-3 \mathrm{~mol} \%$, de composition en $\mathrm{GeH}_{4}, 0-100 \mathrm{~mol} \%$ vis-à-vis du silane. Dans tous les cas, les résultats ont donné des valeurs identiques pour $X$ et $Y$, les hydrures étant complètement dissociés lorsque l'équilibre est atteint sans donner de sous-produits gazeux de silicium ou de germanium. La composition initiale du gaz en germane et en silane fixe ainsi la stœechiométrie du dépôt.

La situation est différente lorsque des espèces gazeuses plus stables sont introduites dans le réacteur. La figure 2 représente l'évolution de la grandeur $X$ en fonction de la température, pour les deux systèmes chimiques réactifs simulés : $89 \% \mathrm{Ar}-1 \% \mathrm{GeH}_{4}+\mathrm{SiH}_{4}-9,96 \% \mathrm{H}_{2}-$ $0,04 \% \mathrm{HCl}$ et $99 \% \mathrm{Ar}-1 \% \mathrm{GeCl}_{4}+\mathrm{SiH}_{4}$. Pour le premier, le rapport $\mathrm{Cl} / \mathrm{H}$ fixé est très faible $\left(0,85 \times 10^{-3}\right)$ compte tenu de la dilution de $\mathrm{HCl}$ utilisée. Pour le second, le rapport $\mathrm{Cl} / \mathrm{H}$ varie 


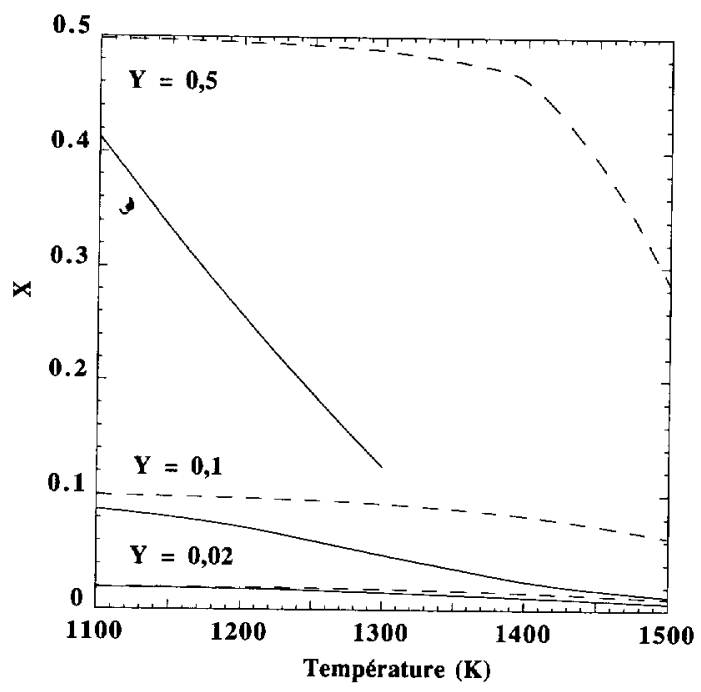

Fig. 2. - Evolution de $X$ en fonction de la température pour différents mélanges gazeux à $P=315$ Pa. ( - ) : Ar $(99 \%)-\mathrm{SiH}_{4}+\mathrm{GeCl}_{4}(1 \%) ; \mathrm{Cl} / \mathrm{H}=Y_{\mathrm{Ge}} /\left(1-Y_{\mathrm{Ge}}\right)(\ldots-\ldots): \operatorname{Ar}(89 \%)-\mathrm{SiH}_{4}+$ $\mathrm{GeH}_{4}(1 \%)-\mathrm{H}_{2}(9,96 \%)+\mathrm{HCl}(0,04 \%) \mathrm{Cl} / \mathrm{H}=0,85 \times 10^{-3}$.

[Evolution of $X$ as a function of the temperature for different mixtures at $P=315 \mathrm{~Pa}$. ( - ): Ar $(99 \%)-\mathrm{SiH}_{4}+\mathrm{GeCl}_{4}(1 \%) ; \mathrm{Cl} / \mathrm{H}=Y /(1-Y)(---)$ : $\mathrm{Ar}(89 \%)-\mathrm{SiH}_{4}+\mathrm{GeH}_{4}(1 \%)-\mathrm{H}_{2}(9.96 \%)$ $+\mathrm{HCl}(0.04 \%) ; \mathrm{Cl} / \mathrm{H}=0.85 \times 10^{-3}$ ]

avec la quantité de germanium introduite :

$$
\mathrm{Cl} / \mathrm{H}=\frac{Y}{1-Y}
$$

A la pression réduite utilisée dans nos expériences, $P=315 \mathrm{~Pa}$, la présence de chlore provoque une diminution de la fraction $X$ qui est d'autant plus importante que la température est élévée et que la valeur $Y$ est élevée. Dans tous les cas, le dépôt s'appauvrit en germanium lorsque la température augmente. Ce rôle de la température est d'autant plus important que la quantité de chlore introduite est grande. Les hydrures n'ont pas d'influence sur la composition du dépôt car ils sont entièrement décomposés.

En conclusion, la simulation thermodynamique des phénomènes réactifs à l'équilibre revèle une dépendance de la quantité de germanium dans la couche de $\mathrm{Si}_{1-x} \mathrm{Ge}_{\boldsymbol{x}}$ avec la quantité de chlore, donc avec le rapport $\mathrm{Cl} / \mathrm{H}$, dans le mélange initial, ceci à la pression de $315 \mathrm{~Pa}$ fixée pour les expériences préliminaires qui vont être présentées. Il est cependant important de rappeler que la pression totale, la dilution des réactifs et la nature du gaz porteur peuvent modifier largement les résultats obtenus [6]. Les calculs effectués sur le système $\mathrm{GeCl}_{4}-\mathrm{SiH}_{4}-\mathrm{Ar}$ ont seulement pour but de valider les données thermodynamiques et de démontrer l'importance du chlore pour des applications futures.

\section{Résultats expérimentaux}

Des études antérieures $[3,10,11]$ montrent que pour les différents mélanges réactifs gazeux dilués dans un gaz porteur conduisant au dépôt de $\mathrm{Si}_{1-x} \mathrm{Ge}_{x}$ (a) dans le domaine basse température ( $<1000 \mathrm{~K}$ environ), la vitesse de dépôt croît avec la température et qu'elle est contrôlée par 

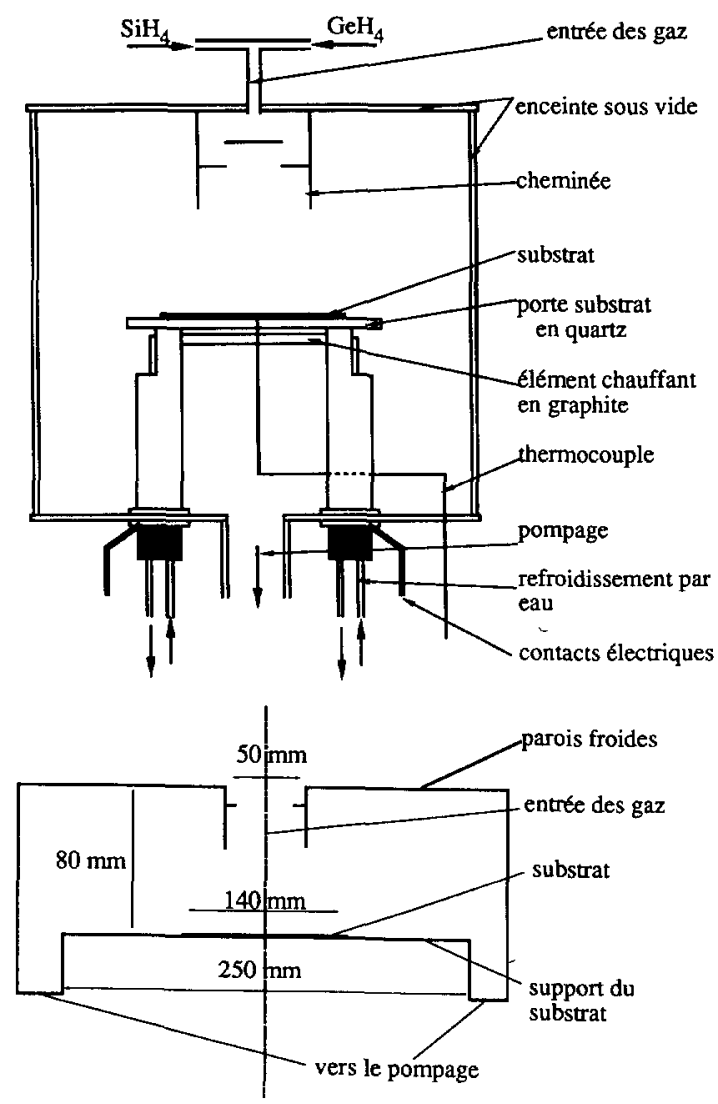

Fig. 3. - Représentation schématique du réacteur.

[Schematic representation of the reactor.]

des phénomènes cinétiques hétérogènes, (b) dans le domaine haute température, la vitesse de dépôt est contrôlée par le transfert de matière réactive de la phase gazeuse vers la surface du substrat. Des observations plus spécifiques ont montré qu'à basse pression ( $800 \mathrm{~Pa})$ et à basse température $(973 \mathrm{~K}$ ) lorsque le régime limitant est le régime chimique, la vitesse de dépôt de $\mathrm{Si}_{1-x} \mathrm{Ge}_{x}$ à partir de germane $\left(\mathrm{GeH}_{4}\right)$ et de dichlorosilane $\left(\mathrm{SiH}_{2} \mathrm{Cl}_{2}\right)$ est plus rapide (environ $0,05 \mu \mathrm{m} \cdot \mathrm{mn}^{-1}$ ) que celle du silicium à partir de $\mathrm{SiH}_{2} \mathrm{Cl}_{2}$ (environ $0,02 \mu \mathrm{m} \cdot \mathrm{mn}^{-1}$ ) [10]. Le même phénomène a été observé à pression atmosphérique et à plus haute température (1173 $\mathrm{K})$ lorsque le régime limitant est le transfert de matière [11]. Lorsque $\mathrm{GeH}_{4}, \mathrm{SiH}_{4}$ et $\mathrm{HCl}$ sont les gaz réactifs introduits dans le réacteur, la vitesse de dépôt de $\mathrm{Si}_{1-x} \mathrm{Ge}_{x}$ en fonction de la température est identique à celle du silicium à partir de $\mathrm{SiH}_{4}$ [12]. En extrapolant les données de la littérature $[10,11]$ pour nos conditions expérimentales $(T=1300 \mathrm{~K}, P=315 \mathrm{~Pa})$, la vitesse de dépôt moyenne tendrait vers $1 \mu \mathrm{m} \cdot \mathrm{mn}^{-1}$ et le régime diffusionnel serait atteint.

Dans le réacteur à parois froides utilisé, le mélange réactif s'écoule d'une entrée froide vers un substrat chauffé à $1300 \mathrm{~K}$; la pression totale est de $315 \mathrm{~Pa}$. Un substrat de $100 \mathrm{~mm}$ de diamètre est posé sur un support de quartz de $250 \mathrm{~mm}$ de diamètre. Le réacteur est en acier inoxydable ( $300 \mathrm{~mm}$ de diamètre et $300 \mathrm{~mm}$ de hauteur). Le substrat est chauffé par un résistor de graphite (Fig. 3). 


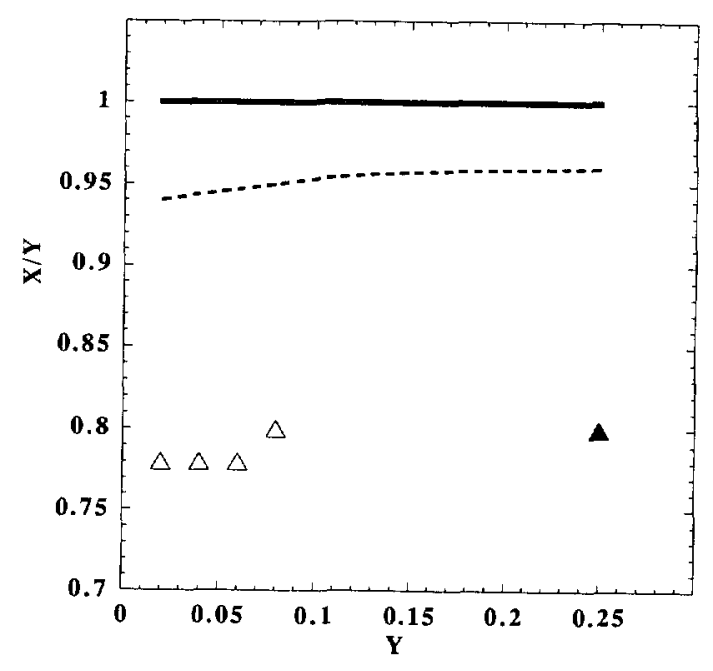

Fig. 4. - Evolution de $X / Y$ en fonction de $Y$ à $1300 \mathrm{~K}$ et $315 \mathrm{~Pa}$. ( - ) : calculs d'équilibre pour le mélange (1): Ar (99\%) - $\mathrm{SiH}_{4}+\mathrm{GeH}_{4}(1 \%) ;(-\ldots-)$ : calculs d'équilibre pour le mélange (2) : $\mathrm{Ar}(89 \%)-\mathrm{SiH}_{4}+\mathrm{GeH}_{4}(1 \%)-\mathrm{H}_{2}(9,96 \%)-\mathrm{HCl}(0,04 \%) ;(\Lambda)$ : expérience avec le mélange 1 , $(\Delta)$ : expériences avec le mélange 2.

[Evolution of $X / Y$ as a function of $Y$ at $1300 \mathrm{~K}$ and $315 \mathrm{~Pa}$ ( - ): equilibrium calculation for the mixture (1): Ar (99\%) - $\mathrm{SiH}_{4}+\mathrm{GeH}_{4}(1 \%) ;(-\cdots)$ : equilibrium calculation for the mixture (2): $\mathrm{Ar}$ $(89 \%)-\mathrm{SiH}_{4}+\mathrm{GeH}_{4}(1 \%)-\mathrm{H}_{2}(9.96 \%)-\mathrm{HCl}(0.04 \%) ;(\Delta)$ : experiments with the mixture $1,(\Delta)$ : experiments with the mixture 2.]

L'addition de petites quantités de $\mathrm{HCl}(0,04 \%)$ au mélange $\mathrm{Ar}(89 \%)-\mathrm{H}_{2}(9,96 \%)-\mathrm{SiH}_{4}$ $+\mathrm{GeH}_{4}(1 \%)$ à $315 \mathrm{~Pa}$ et à $1300 \mathrm{~K}$ conduit à une valeur de $X$ variant de 0,015 à 0,064 lorsque $Y$ varie de 0,02 à 0,08 et à un rapport $X / Y$ sensiblement constant et de l'ordre de 0,77-0,8 (Fig. 4). L'analyse thermodynamique seule ne permet pas de retrouver les valeurs de ce rapport.

Dans une autre expérience effectuée sans addition de $\mathrm{HCl}$, avec $Y=0,25$ (mélange $\mathrm{Ar}(99 \%)$, $\left.\mathrm{SiH}_{4}(0,75 \%), \mathrm{GeH}_{4}(0,25 \%)\right)$ une fraction de germanium, $X$, de 0,20 dans le solide a été obtenue alors que l'analyse thermodynamique, faite à partir du mélange gazeux initial prédisait $X=Y=0,25$. Ces valeurs ont été mesurées au centre du substrat. Une augmentation de l'ordre de $10 \%$ est observée entre le centre et la périphérie du substrat. La vitesse de croissance diminue de $1,4 \mu \mathrm{m} \cdot \mathrm{mn}^{-1}$ au centre à $0,4 \mu \mathrm{m} \cdot \mathrm{mn}^{-1}$ à la périphérie du substrat. La courbe en trait plein de la figure 4 résulte de l'analyse thermodynamique lorsque les réactifs ne contiennent pas de chlore. Elle met en évidence que le rendement thermodynamique est de $100 \%$. La courbe en trait pointillé montre que lorsque de faibles quantités de $\mathrm{HCl}$ sont introduites, le rendement est plus faible du fait de la plus grande stabilité des chlorures. Les stœchiométries expérimentales obtenues en présence ou en l'absence de $\mathrm{HCl}$ ne concordent pas avec celles prédites par le calcul thermodynamique à partir des conditions initiales. Ceci indique, que la part des phénomènes de transfert de matière (convection, diffusion, thermodiffusion) et de cinétique réactionnelle doit être pris en compte au cours de l'élaboration du dépôt. 
Tableau III. - Equations décrivant les phénomènes de transfert.

[The equations describing transport phenomena.]

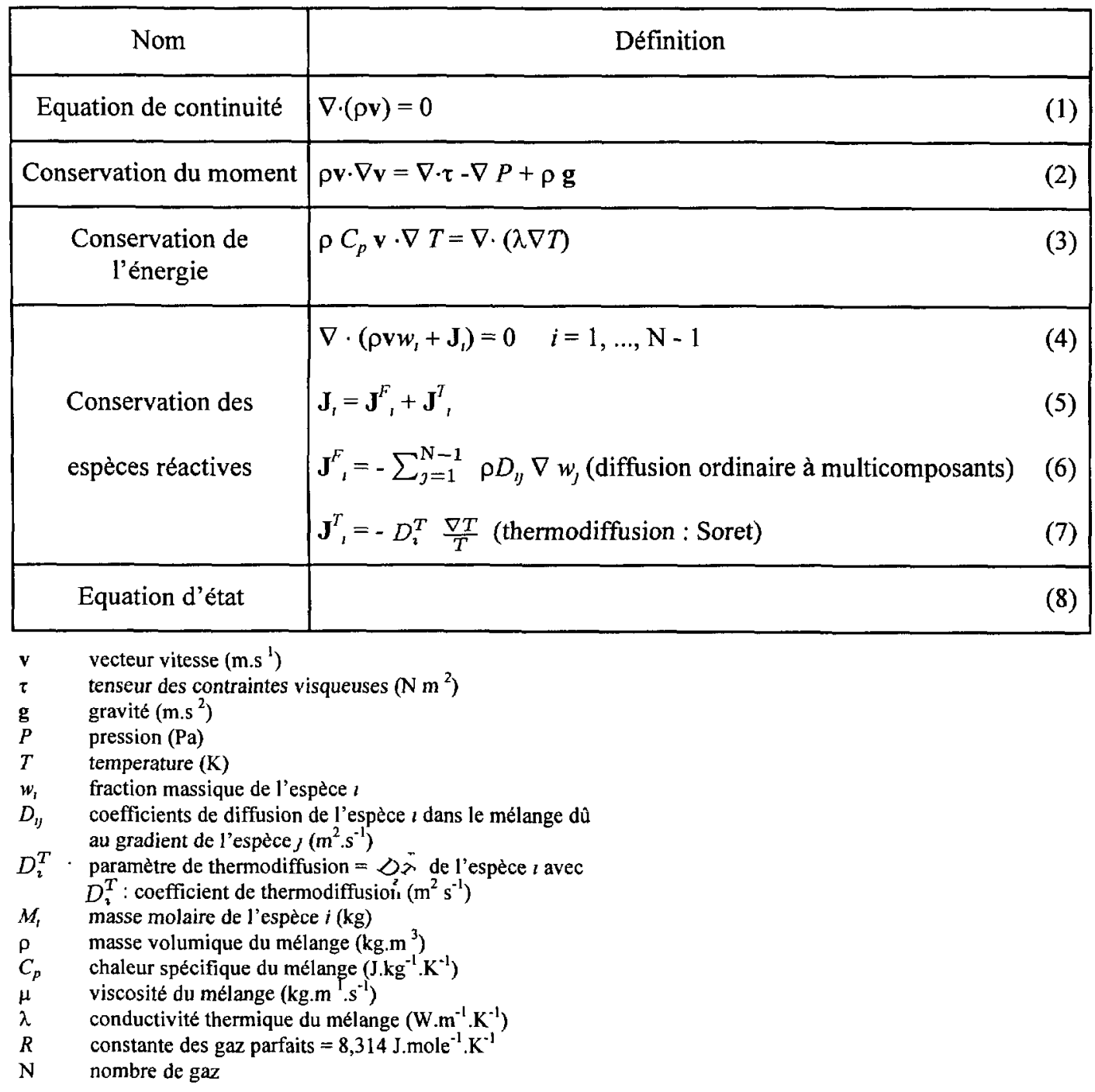

\section{Analyse des phénomènes de transfert de matière}

4.1. Modélisation. - L'objectif de cette étude est d'analyser l'influence des phénomènes de transfert de matière sur la croissance du matériau à partir du mélange $\mathrm{Ar}-\mathrm{SiH}_{4}-\mathrm{GeH}_{4}$. Cette modélisation repose sur les hypothèses simplificatrices suivantes : on admet qu'il n'y a pas de réactions chimiques homogènes dans la phase gazeuse et que l'équilibre thermodynamique est atteint au cours des réactions gaz-solide à la surface du substrat. A I'heure actuelle, il semble difficile de proposer une modélisation plus avancée de la croissance de $\mathrm{Si}_{1-x} \mathrm{Ge}_{\boldsymbol{x}}$ par CVD.

Les équations différentielles décrivant les phénomènes de transfert survenant lors de l'élaboration du dépôt sont présentées dans le tableau III [13-18]. Le modèle proposé suppose que l'écoulement gazeux est stationnaire et laminaire et que la loi des gaz parfaits est applicable. Le 
schéma du réacteur bidimensionnel axisymétrique à parois refroidies utilisé pour les simulations est représenté sur la figure 3 .

Les conditions aux limites associées aux équations 1-8 pour le réacteur expérimental et pour le mélange $\mathrm{Ar}-\mathrm{SiH}_{4}-\mathrm{GeH}_{4}$ sont les suivantes :

a) la vitesse est fixée à l'entrée,

b) la vitesse est nulle sur les parois,

c) les températures des parois sont connues,

d) les fractions massiques des espèces gazeuses sont connues à l'entrée,

e) les flux des espèces gazeuses sont nuls sur les parois non réactives,

f) les fractions massiques des espèces gazeuses sur le substrat sont nulles (négligeables à l'équilibre thermodynamique).

Les coefficients de transfert $\lambda, \mu, D_{\imath \jmath}, D_{\imath}^{T}$ sont estimées à partir de la théorie cinétique des gaz [19-22]. Les coefficients de diffusion à multicomposants et les coefficients de thermodiffusion ont été calculés à l'aide de transformations matricielles [22,23]. Les propriétés du mélange ont été lissées par des polynômes du second degré pour chaque inconnue, la température et les fractions massiques des gaz réactifs. Enfin, nous avons utilisé l'extension CVD du logiciel général de type éléments finis Flux-Expert [24] présentée précédemment [23]. Pour cette simulation 2D, un maillage d'environ 2000 nœuds a été utilisé. La convergence est atteinte en 6 heures sur une station Sun Sparc 2.

\subsection{Application du modèle au Cas du mélange Ar (99\%), $\mathrm{SiH}_{4}+\mathrm{GeH}_{4}(1 \%)$}

Les parois du réacteur sont refroidies $(T=300 \mathrm{~K})$ et le substrat est chauffé $(T=1300$ $\mathrm{K})$. La vitesse du mélange gazeux est connue à l'entrée du réacteur et son profil est supposé parabolique. Les conditions expérimentales standards sont caractérisées par un débit total de 2 L. $\mathrm{mn}^{-1}$, une pression totale de $315 \mathrm{~Pa}$ et des fractions molaires de $\mathrm{SiH}_{4}$ et $\mathrm{GeH}_{4}$ dilués dans l'argon à $0,85 \%$ et $0,15 \%(Y=0,15)$.

Les champs de vitesse, de température et de concentrations obtenus dans ces conditions expérimentales standards, sont tracés sur la figure 5 . La forme générale de ces champs est très peu modifiée lorsque les proportions relatives de $\mathrm{SiH}_{4}$ et $\mathrm{GeH}_{4}$ varient (de 0 à $1 \%$ ). Le calcul permet de déterminer les flux de $\mathrm{SiH}_{4}$ et de $\mathrm{GeH}_{4}$ normaux au substrat et ainsi la vitesse de dépôt et la valeur de $X$ d'après :

$$
X=\frac{J_{\mathrm{GeH}_{4}}}{J_{\mathrm{GeH}_{4}}+J_{\mathrm{SiH}_{4}}}
$$

Les flux de $\mathrm{SiH}_{4}$ et de $\mathrm{GeH}_{4}$, donc la vitesse de dépôt de $\mathrm{Si}_{1-x} \mathrm{Ge}_{x}$, varient radialement le long du substrat du fait de l'importance relative des flux de matière et de la consommation des gaz réactifs sur le substrat (Fig. 6a). Toutefois les valeurs de $X$ restent pratiquement constantes et de l'ordre de $0,12-0,14$ alors que $Y=0,15$. La vitesse de dépôt de la solution solide $\operatorname{Si}_{1-x} \mathrm{Ge}_{x}$ (Fig. 6c) est calculée à partir des flux de silane et de germane et de la stœchiométrie du dépôt (Fig. 6b). On peut noter que la thermodiffusion a pour effet d'appauvrir les régions chaudes en composé le plus lourd, $\mathrm{GeH}_{4}$ et ainsi de diminuer la quantité de germanium dans le dépôt. Cet effet est du deuxième ordre car, l'argon étant utilisé comme gaz porteur, les différences de masse sont petites. Cet effet serait plus important si l'hydrogène était le gaz porteur. Les vitesses de dépôt calculées varient plus fortement (de $1,7 \mu \mathrm{m} \cdot \mathrm{mn}^{-1}$ à $0,2 \mu \mathrm{m} \cdot \mathrm{mn}^{-1}$ ) le long du substrat 


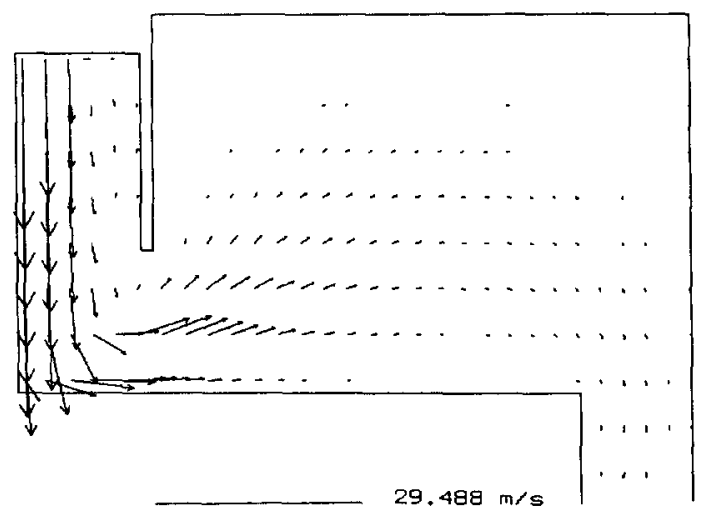

a)

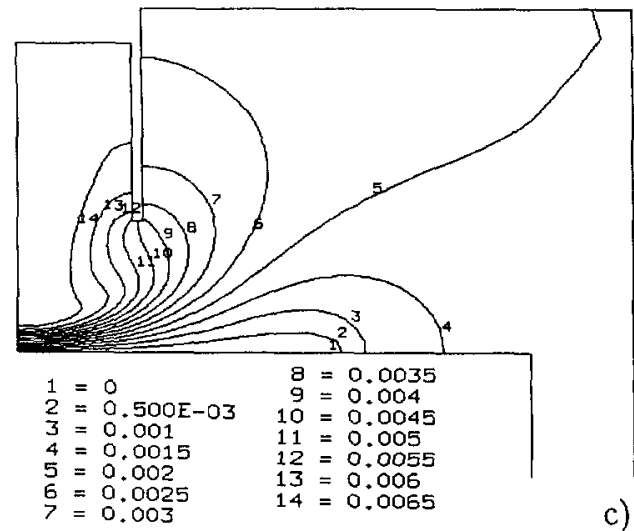

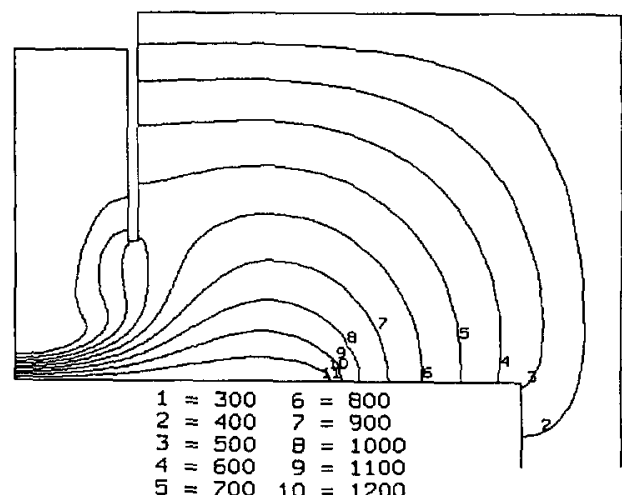

b)

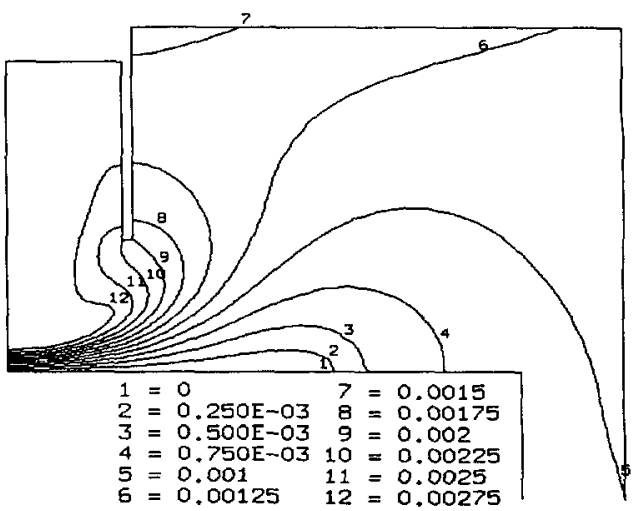

d)

Fig. 5. - Champs de (a) vitesse, (b) température, (c) fraction massique de $\mathrm{SiH}_{4}$, (d) fraction massique de $\mathrm{GeH}_{4}$ calculés pour le réacteur de la figure 3 et pour les conditions expérimentales suivantes : Débit total : $2 \mathrm{l} / \mathrm{mn}$ de $\mathrm{Ar}(99 \%)-\mathrm{SiH}_{4}(0,85 \%)-\mathrm{GeH}_{4}(0,15 \%)(Y=0,15)$; température du substrat $1300 \mathrm{~K}$; pression totale $315 \mathrm{~Pa}$. Seule la partie droite du réacteur est présentée.

[(a) velocity (b) temperature, (c) mass fraction of $\mathrm{SiH}_{4}$, (d) mass fraction of $\mathrm{GeH}_{4}$ computed for the geometry of figure 3 and for experimental conditions: Total flow rate: $2 \mathrm{l} / \mathrm{mn}$ de $\mathrm{Ar}(99 \%)-\mathrm{SiH}_{4}$ $(0.85 \%)-\mathrm{GeH}_{4}(0.15 \%)(Y=0.15)$; Substrate temperature $1300 \mathrm{~K}$; Total pressure $315 \mathrm{~Pa}$. Only half right part of the reactor is presented.]

que les vitesses de dépôt expérimentales bien qu'elles restent du même ordre de grandeur. Ceci provient très certainement des conditions aux limites extrêmes sur le substrat mais surtout d'un modèle chimique trop simplifié dont nous discuterons par la suite. Expérimentalement, les vitesses de dépôts moyennes sont de l'ordre de $1 \mu \mathrm{m} . \mathrm{mn}^{-1}$ et peuvent varier d'un facteur 3 entre le centre et la périphérie du substrat lorsque celui-ci atteint des dimensions importantes $(10 \mathrm{~cm})$.

Les différents flux (convection, diffusion et thermodiffusion) sont représentés sur la figure 7 le long de l'axe du réacteur. Au voisinage de l'axe de symétrie, les composantes radiales des différents flux sont pratiquement nulles. Les phénomènes de transfert sont alors quasiment mono-dimensionnels le long de l'axe du réacteur. Les flux de convection liés à la vitesse barycentrique du mélange diminuent lorsque l'on s'approche du substrat alors que les flux de diffusion augmentent (en valeur absolue) du fait de la consommation du silane et du germane 


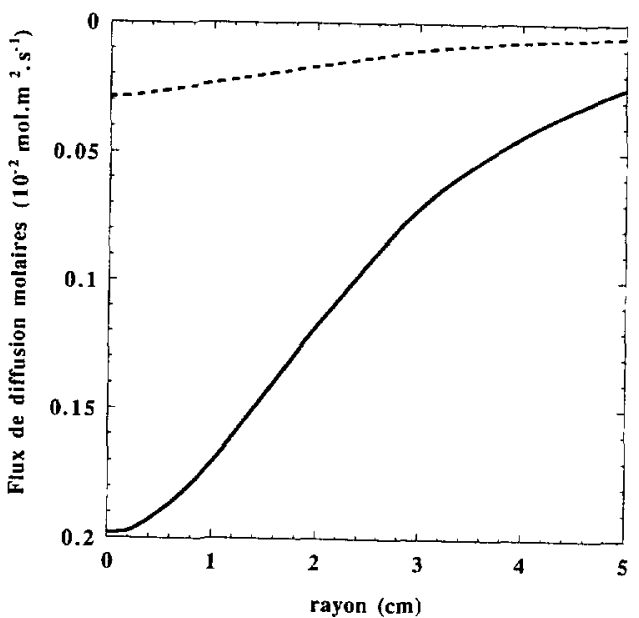

a)

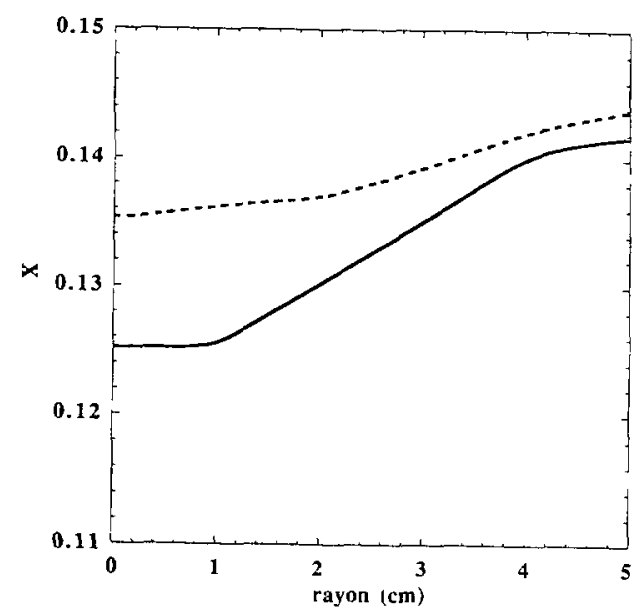

b)

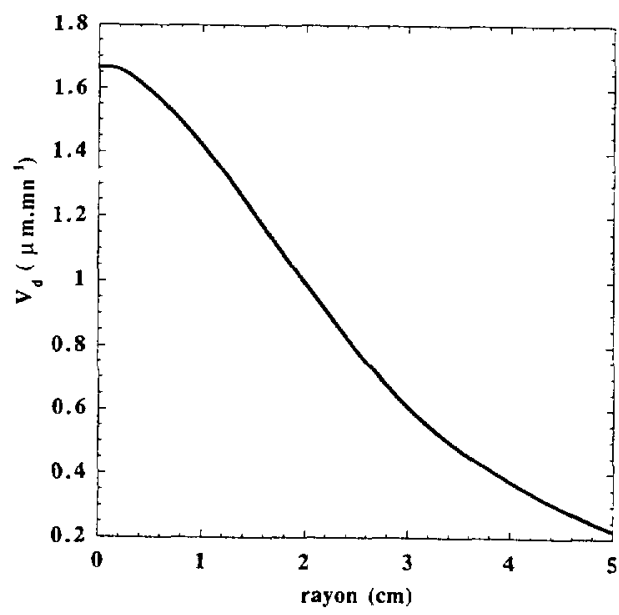

c)

Fig. 6. - (a) Flux molaires (composante verticale) de silane ( - ) et de germane ( - _ - ) calculés à la surface du substrat ; (b) Stœchiométrie calculée du revêtement le long du substrat avec ( - ) et sans ( - - ) prise en compte de la thermodiffusion ; (c) Vitesse de dépôt de $\mathrm{Si}_{1-x} \mathrm{Ge}_{x}$ (en $\mu \mathrm{m} \mathrm{mn}{ }^{-1}$ ) le long du substrat. Les conditions expérimentales sont celles décrites sur la figure 5.

[(a) Molar fluxes (axial) of silane ( - ) and of germane ( - . - ) computed at the substrate surface. (b) Computed stoichiometry of the coating along the substrate with (-) and without (--) thermodiffusion. (c) Deposition $\left(\mu \mathrm{m} \cdot \mathrm{mn}^{-1}\right)$ rate along the substrate. Experimental conditions are those mentioned in figure 5.]

qui provoque des gradients de concentrations importants. Quant aux flux de thermodiffusion du silane et du germane, ils sont opposés du fait de leurs comportements respectifs vis-à-vis du gaz porteur et du champ de température. Alors que les rapports des flux de convection et de diffusion du germane et du silane sont voisins du rapport des quantités de silane et de germane introduites dans le réacteur, le rapport des flux de thermodiffusion est très différent de cette valeur et, bien que ces flux soient beaucoup plus petits que les flux de diffusion, leur influence 


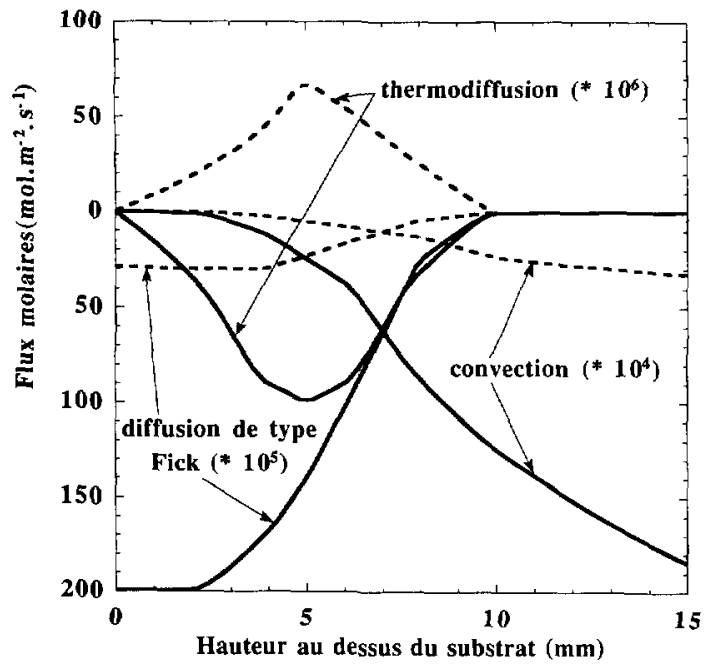

Fig. 7

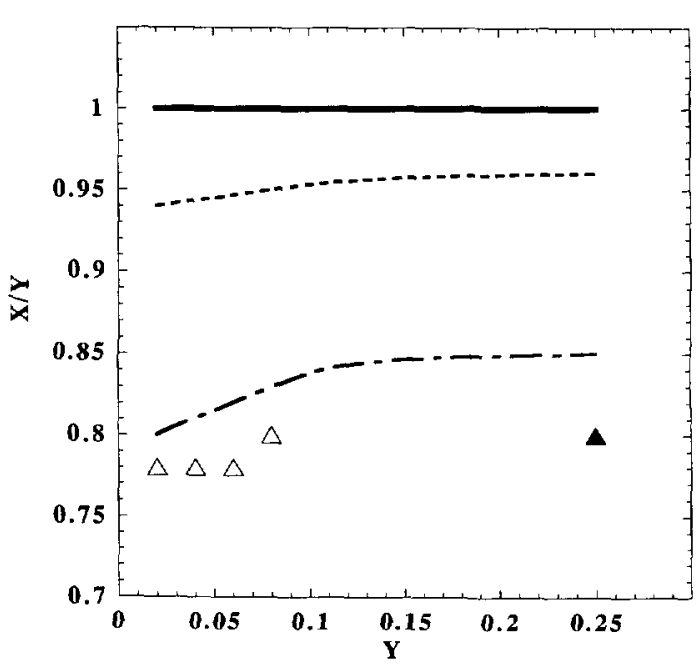

Fig. 8

Fig. 7. - (a) Les différents flux molaires (composante verticale) de silane ( - ) et de germane ( - ). Les conditions expérimentales sont celles décrites sur la figure 5 .

[(a) Different molar fluxes (axial) of silane ( - ) and of germane ( - - Experimental conditions are those mentioned in figure 5 .

Fig, 8. - Evolution de $X / Y$ en fonction de $Y$ à $1300 \mathrm{~K}$ et $315 \mathrm{~Pa}$ pour les mélanges (1) et (2) définis dans la figure $4 .(\ldots)$ : analyse thermodynamique en l'absence de chlore (mélange 1$) ;(\ldots .$.$) :$ analyse thermodynamique en présence de chlore (mélange 2) ; $\left.--{ }_{-}\right)$: analyse du transfert de matière pour le mélange $1 ;(\boldsymbol{\Lambda})$ expériences avec le mélánge $1 ;(\Delta)$ expériences avec le mélange 2 .

[Evolution of $X / Y$ as a function of $Y$ at $1300 \mathrm{~K}$ and $315 \mathrm{~Pa}$ for the mixtures (1) et (2) defined in figure 4. ( - ) : thermodynamic analysis without chlorine (mixture 1$) ;(\ldots-)$ : thermodynamic analysis with chlorine (mixture 2); ( - - - ) : mass transport analysis for mixture $1 ;(\mathbf{A})$ experiments with mixture $1 ;(\Delta)$ experiments with mixture 2.]

sur la stœchiométrie du dépôt (appauvrissement en germanium) n'est pas négligeable.

Les résultats des simulations réalisées pour différentes valeurs de $Y$ sont représentées sur la figure 8. Les valeurs représentées sont celles calculées et mesurées au centre du substrat. On remarque que la prise en compte du transfert de matière est nécessaire. Le modèle de transfert, extrêmement simplifié, devrait être amélioré par la prise en compte des cinétiques de réactions homogènes et hétérogènes mais, à l'heure actuelle, les données ne sont pas disponibles. Par contre, il est possible de coupler les calculs thermodynamiques et de transfert de matière afin de simuler de façon plus précise la réactivité chimique à l'équilibre $[25,26]$. Mais cette approche serait encore trop simplifiée pour traduire tous les phénomènes chimiques observés dans des travaux récents, notamment pour le dépôt de silicium à partir des systèmes $\mathrm{H}_{2}-\mathrm{SiH}_{4}$ [27-29] et $\mathrm{H}_{2}-\mathrm{Si}_{2} \mathrm{H}_{6}[30]$. Ces études ont été centrées sur l'influence des réactions homogènes et hétérogènes lors de la croissance du dépôt de silicium à partir du silane. Des modèles cinétiques prenant en compte jusqu'à 31 réactions homogènes ont ainsi permis de montrer que les espèces intermédiaires (en particulier $\mathrm{SiH}_{2}$ et $\mathrm{Si}_{2} \mathrm{H}_{6}$ ) jouaient un rôle important lors de l'élaboration du dépôt. Les espèces radicalaires telle que $\mathrm{SiH}_{2}$ sont très réactives et les travaux de Coltrin $e t$ al. [27] leur attribuent un coefficient de collage (probabilité de réaction) de l'ordre de 1 alors que celui du silane serait de l'ordre de $10^{-5}$ A basse température, les réactions en phase gazeuse 
sont négligeables ; l'élaboration du dépôt est alors contrôlée par la cinétique hétérogène de décomposition du silane. Par contre, à haute température (>1200 K), cas de notre étude, la décomposition homogène du silane devient importante [29]. La vitesse de dépôt augmente si la température augmente car le cœefficient de collage du $\mathrm{SiH}_{4}$ augmente mais aussi, et surtout, du fait de la croissance exponentielle de la concentration d'espèces intermédiaires très réactives. A haute température, les modèles cinétiques tendent à montrer que l'équilibre homogène est atteint. Par contre, compte tenu du faible cœefficient de collage du silane résiduel, l'équilibre hétérogène ne serait pas atteint. Seules les espèces ayant un cœefficient de collage voisin de 1 pourraient jouer un rôle dans l'équilibre gaz-solide. De plus, l'équilibre suppose que le temps de séjour dans le réacteur est suffisamment long. Les travaux récents de Wang et al. [30] ont montré que certaines espèces, en particulier à pression réduite, n'avaient pas le temps de se former. Pour le système Ge-H ce type d'information n'est pas disponible actuellement mais on peut supposer une similitude de comportement entre les systèmes Ge-H et Si-H.

$\mathrm{Au} \mathrm{vu} \mathrm{de} \mathrm{cet} \mathrm{aperçu} \mathrm{rapide,} \mathrm{il} \mathrm{semble} \mathrm{que} \mathrm{les} \mathrm{équilibres} \mathrm{homogène} \mathrm{et} \mathrm{hétérogène} \mathrm{ne} \mathrm{sont}$ pas simultanément atteints dans un réacteur où le volume de gaz porté à la température du substrat a des dimensions restreintes.

Dans l'état actuel des connaissances, il semble clair que la croissance de $\mathrm{Si}_{1-x} \mathrm{Ge}_{x}$ ne peut être interprétée que sur la base de mécanismes transfert-réaction. Le modèle de transfert de matière simplifié proposé montre que la vitesse de dépôt, son uniformité et la stcechiométrie du matériau déposé sont influencées par les phénomènes de transfert. En ce qui concerne la cinétique, un effort expérimental important devrait être fait afin d'obtenir des données précises concernant la réactivité.

\section{Conclusion}

Les simulations décrivant la croissance de $\mathrm{Si}_{1-x} \mathrm{Ge}_{x}$ à partir du $\mathrm{Ar}-\mathrm{SiH}_{4}+\mathrm{GeH}_{4}$ montrent que l'analyse thermodynamique ne permet pas seule d'interpréter les mesures de composition du solide et que les phénomènes de transfert de matière en phase gazeuse jouent un rôle important sur la vitesse de dépôt et la stœechiométrie du matériau déposé. Cette analyse a mis en évidence le fait que la quantité de germanium dans la couche est toujours inférieure à la quantité de germanium introduite sous forme de germane. Cet appauvrissement en germane au voisinage du substrat est amplifié par le phénomène de transfert du gaz réactif par thermodiffusion. Le bilan de cette analyse est bien sûr moins riche que ce que pourrait apporter l'analyse thermodynamique des équilibres homogène et hétérogène couplée au transfert de matière. Cependant, elle apporte un complément d'information sur la nature dynamique du procédé. De plus, si l'équilibre thermodynamique hétérogène prévoit que les hydrures de silicium et de germanium sont totalement dissociés, il n'en est pas de même pour leurs chlorures. Dans l'avenir, afin d'obtenir des informations sur la réactivité à l'équilibre pour des réactifs gazeux chlorés, un couplage équilibre-transfert s'avère indispensable. 


\section{Bibliographie}

[1] Fujanaga K., et Karasawa T., Characteristics of B-doped $\mathrm{Si}_{1-x} \mathrm{Ge}_{x}$ growth rates by chemical vapor deposition using $\mathrm{Si}_{2} \mathrm{H}_{6}, \mathrm{GeH}_{4}$ and $\mathrm{B}_{2} \mathrm{H}_{6}$ gases, Surf. Coat. Technol. 140 (1993) 2081-2084.

[2] Holleman J., Kuiper A.E.T. et Verweij J.F., Kinetics of the low pressure chemical vapor deposition of polycristalline germanium-silicon alloys from $\mathrm{SiH}_{4}$ and $\mathrm{GeH}_{4}, J$. Electrochem. Soc. 140 (1993) 1717-1722.

[3] Madar R., Mastromatteo E., Magerl A., Liss K.D. et Bernard C., Low pressure chemical vapor deposition of massive $\mathrm{Si}_{1-x} \mathrm{Ge}_{x}$ gradient crystals and applications in short-wavelength diffraction, Surf. Coat. Technol. 54/55 (1992) 229-233.

[4] Barbier J.N. et Bernard C., "Melange software package", Proceedings of the 15th Calphad Meeting (B.L. Kaufman Ed., Calphad, 1986) 206-212.

[5] SGTE databank, Scientific Group Thermodata Europe, BP 66, 38402 Saint-Martin d'Hères, France.

[6] Rouch H., Modélisation du procédé de dépôt chimique à partir d'une phase vapeur, Thèse Institut National Polytechnique de Grenoble (1994).

[7] Bergman C., Chastel R. et Castanet R., Thermodynamic investigation on the Si-Ge binary system by calorimetry and Knudsen cell mass spectrometry, J. Phase Equi. 13 (1992) 113-118.

[8] Chang S., Unizicker D. et Anderson T.J., Thermodynamic analysis of $\mathrm{Si}_{x} \mathrm{Ge}_{1-x}$ chemical vapor deposition, Proceeding of the 10th CVD International Conference 1987, G.W. Cullen Ed., The Electrochemical Society (Pennington, NJ, 1987) pp. 122-131.

[9] Tang H.P., Vescan L. et Lüth H., Thermodynamic analysis of SixGe1-x chemical vapor deposition, J. Crystal Growth 116 (1992) 301-310.

[10] Garone P. M., Sturm J. C., Schwartz P. V., Schwartz S.A. et Wilkens B., Silicon vapor phase epitaxial growth catalysis by the presence of germane, Appl. Phys. Lett. 56 (1990) 1275-1277.

[11] Kühne H. et Richter H., Transport-reaction controlled chemical vapor deposition of epitaxial $\mathrm{Si}_{1-x} \mathrm{Ge}_{x}$ thin films and thermodynamic equilibrium composition, J. Mater. Res. 8 (1993) 131137.

[12] De Boer W.B. et Meyer D., Low temperature chemical vapor deposition of epitaxial Si and SiGe layers at atmospheric pressure, J. Appl. Phys. Lett. 58 (1991) 1286-1288.

[13] Jensen K.F. et Kern W., Thermal chemical vapor deposition, Thin Film Processes II, J.L. Vossen and W. Kern Eds. (Academic Press, 1991) pp. 283-368.

[14] Holstein W.L., Design and modeling of chemical vapor deposition reactors, Prog. Cryst. Growth Charact. 24 (1992) 111-211.

[15] Gokuglu S.A., Chemical vapor deposition for high temperature materials, Mat. Res. Soc. Symp. Proc. 250 (1992) 17-28.

[16] Kleijn C.R. et Werner C., Modelling of Chemical Vapor Deposition of Tungsten Films, K. Merten et A. Gilg Eds. (Birkhaüser, Verlag, Basel, 1993).

[17] Pons M., Klein R., Arena C. et Mariaux S., Modeling of cold wall chemical vapor deposition reactors, J. Phys. C5 (50) (1989) 57.

[18] Jensen K.F., Chemical vapor deposition, Microelectronic Processing, D.W. Hess et K.F. Jensen Eds. (American Chemical Society, Washington D.C., 1989) pp. 199-263.

[19] Kleijn C.R., Transport phenomena in CVD reactors, $\mathrm{PhD}$ Thesis, Delf University (1991).

[20] Bird R.B., Stewart W.E. et Lightfoot E.N., Transport Phenomena (Wiley , New York, 1960).

[21] Hirchfelder J.O., Curtiss C.F. et Bird R.B., Molecular Theory of Gases and Liquids (John Wiley, New York, 1967).

[22] Cussler E.L., Diffusion Mass Transfer in Fluid Systems (Cambridge University press, 1984).

[23] Pons M., Benezech A., Huguet P., Gaufres R. et Diez Ph., Mass transport modeling during tungsten chemical vapor deposition, J.C.V.D., sous presse. 
[24] Flux-expert, Société DT2I, Chemin des Prêles, 38240 Meylan, France.

[25] Pons M., Barbier J.N., Bernard C. et Madar R., A coupled approach to thermochemical and mass transport modeling: application to $\mathrm{TiSi}_{2}$ deposition by C.V.D., Appl. Surf. Sci. 73 (1993) 71-81.

[26] Pons M., Bernard C. et Madar R., Numerical modeling for CVD simulation and process optimization: coupled thermochemical and mass transport approaches, Surf. Coat. Technol. 61 (1993) 274-281.

[27] Coltrin M.E., Keer J. et Evans G.H., A mathematical model of silicon chemical vapor deposition, J. Electrochem. Soc. 130 (1989) 819-829.

[28] Azzaro C., Duverneuil P. et Couderc J.P., Two dimensional modeling of LPCVD hot wall tubular reactors, part II: systematic analysis of pure and phosphorus in situ doped polycristalline silicone depositions, J. Electrochem. Soc. 139 (1992) 305-312.

[29] Wang Y.B., Chaussavoine C. et Teyssandier F., 2D modelling of a non-confined circular impinging jet reactor; Si chemical vapor deposition, J. Crystal Growth 126 (1993) 373-395.

[30] Wang Y.B., Teyssandier F., Simon J. et Feurer R., Chemical vapour deposition of silicon from disilane under reduced pressure in a circular impinging jet reactor. Simulation and experiments, J. Electrochem. Soc. 141 (1994) 824-843. 